\title{
Ein stochastisches Modell zur Beschreibung von Signalen in digitalen Schaltungen basierend auf quadratischer Optimierung
}

\author{
V. B. Kleeberger, P. Maier, and U. Schlichtmann \\ Lehrstuhl für Entwurfsautomatisierung, Technische Universität München, Germany \\ Correspondence to: V. B. Kleeberger (kleeberger@tum.de)
}

\begin{abstract}
Zusammenfassung. Die kontinuierlich fortschreitende Miniaturisierung in integrierten Schaltungen führt zu einem erhöhten Modellierungsbedarf verschiedenster Effekte, wie z.B. Alterung oder Stromverbrauch. Diese hängen von den auftretenden Signalen innerhalb der Schaltung ab, wodurch deren statistische Modellierung ein zentrales Problem darstellt. Dieser Beitrag stellt eine neue Methode zur stochastischen Signalmodellierung basierend auf quadratischer Optimierung vor. Die Methode wird mit Hilfe von realen Daten mit existierenden Ansätzen verglichen. Die Testergebnisse zeigen hierbei im vorgestellten Modell einen Genauigkeitszuwachs von bis zu einem Faktor 10 im Vergleich zu bereits existierenden Modellen.
\end{abstract}

\section{Einleitung}

Ein zentrales Problem beim Entwurf und der Analyse von integrierten Schaltungen ist die Berechnung von Signalwahrscheinlichkeiten innerhalb der Schaltung. Signalwahrscheinlichkeit bezeichnet hierbei im Allgemeinen die Wahrscheinlichkeit, dass ein Signal innerhalb der Schaltung eine bestimmte Eigenschaft besitzt, z.B. die Wahrscheinlichkeit für den Signalwert (d.h. logisch Eins oder Null) oder die Wahrscheinlichkeit für den Signalzustand (d.h. konstant oder schaltend innerhalb eines Taktzyklus).

Der durchschnittliche Stromverbrauch einer Logikzelle kann z.B. über $P_{\mathrm{dyn}}=0.5 C_{L} V_{\mathrm{dd}}^{2} f \alpha$ abgeschätzt werden. Dabei ist $C_{L}$ die Lastkapazität der Zelle, $V_{\mathrm{dd}}$ die Versorgungsspannung, $f$ die Taktfrequenz der Schaltung und $\alpha$ die Wahrscheinlichkeit, dass das Signal am Zellenausgang innerhalb eines Taktzyklus schaltet (Schneider et al., 1996; Najm, 1994). Ausgehend vom Stromverbrauch können außerdem Temperaturunterschiede (Obermeier und Johannes, 2004) und Spannungsabfälle innerhalb der Schaltung (Nassif, 2008) berechnet werden. Weitere Effekte, die von Signalwahrscheinlichkeiten abhängen, sind die Degradation von
Transistorparametern durch Alterung (Lorenz et al., 2009; Najm, 1993), sowie die Maskierungswahrscheinlichkeit von Fehlern (Chen und Tahoori, 2012).

$\mathrm{Da}$ es mit steigender Integrationsdichte zunehmend kostspieliger wird diese Probleme allein mit schaltungstechnischen Maßnahmen zu lösen, gewinnen zunehmend Algorithmen an Bedeutung die es erlauben verschiedene Abstraktionsebenen zu berücksichtigen. Hierzu werden zur Lösung oft zusätzlich Informationen aus der Systemebene berücksichtigt. Die Berechnungen dieser Algorithmen werden wiederum auf System-Ebene zur Verfügung gestellt, um die dort ausgeführten Analysen und Optimierungen genauer und effizienter zu machen. Vor allem im Bereich der Signalmodellierung spielt dies eine entscheidende Rolle, da die anliegenden Signale und damit deren Wahrscheinlichkeiten oft wesentlich von der ausgeführten Software und den verarbeiteten Daten auf dem System abhängen.

Die Berechnung von Signalwahrscheinlichkeiten stellt ein nichttriviales Problem beim Schaltungsentwurf dar. Die einzelnen Signale an den Schaltungseingängen weisen hierbei bereits in der Regel deutliche Korrelationen auf bedingt durch die gegebenen Eingangsdaten. Auch die Schaltungsstruktur selber führt zusätzliche Korrelationen durch ihre Topologie ein. Signalverzweigungen durch Fanouts die später an einem Gatter wieder rekonvergieren führen zu Abhängigkeiten der Gattereingänge, welches die Signalwahrscheinlichkeiten im Vergleich zu unabhängigen Eingängen beeinflusst.

Algorithmen zur Abschätzung von Signalwahrscheinlichkeiten können in zwei Kategorien unterteilt werden. Statistische Algorithmen legen verschiedene Signalwerte an den Schaltungseingängen an und rechnen für jeden Vektor von angelegten Signalwerten die Signalwerte an allen anderen Punkten der Schaltung aus. Mit Hilfe des Gesetzes der großen Zahlen können dann hiermit die Signalwahrscheinlichkeiten abgeschätzt werden. Solche Algorithmen können 
beliebige Verteilungen für die Signale am Schaltungseingang modellieren, berücksichtigen strukturelle Korrelationen der Signale innerhalb der Schaltung und sind damit sehr genau. Da die Schaltung allerdings für eine ausreichend große Zahl von Eingangswerten simuliert werden muss um zuverlässige Abschätzungen zu bekommen, benötigen solche Algorithmen oft sehr lange Laufzeiten.

Stochastische Algorithmen hingegen benutzen analytische Gleichungen um Signalwahrscheinlichkeiten direkt zu berechnen. Solche Algorithmen können wiederum in zwei Unterklassen unterteilt werden. Algorithmen der ersten Unterklasse benutzen binäre Entscheidungsdiagramme (englisch: binary decision diagram, $B D D)$ um die funktionale Abhängigkeit zwischen Signalen innerhalb der Schaltung und deren Eingängen darzustellen (Schneider et al., 1996). Die Schaltungsdarstellung durch BDDs ist vor allem in großen Schaltungen ein Problem, da BDDs im schlimmsten Fall exponentiell mit der Zahl der Schaltungseingänge wachsen. Dies verursacht oft Speicherprobleme bei der Erzeugung dieser BDDs.

Die zweite Unterklasse verwendet Approximationsformeln um Signalwahrscheinlichkeiten iterativ durch die Schaltung zu propagieren (Ercolani et al., 1989; Marculescu et al., 1994; Parker und McCluskey, 1975). Diese Algorithmen weisen gute Laufzeit und akzeptablen Speicherverbrauch auf. Andererseits stellt die erreichbare Genauigkeit durch die verwendeten Approximationen oft ein Problem dar.

Durch die bereits oben erwähnte Wichtigkeit hierarchieübergreifender Ansätze gewinnen Algorithmen zusätzlich an Bedeutung, die hierfür verwendet werden können. Grundlegend notwendige Eigenschaft für solche Ansätze ist vor allem eine ausreichend schnelle Laufzeit um Eigenschaften aus der Schaltungsebene extrahieren und in Analysemethoden auf Systemebene verwenden zu können. Diese Arbeit stellt einen solchen Ansatz vor der sich neben guter Laufzeit vor allem durch eine hohe Genauigkeit auszeichnet.

Im Weiteren führt das Kapitel 2 generelle Definitionen und Eigenschaften von Signalwahrscheinlichkeiten ein die in dieser Arbeit verwendet werden. Kapitel 3 stellt den Stand der Technik in diesem Bereich und die wesentlichen Neuerungen dieser Arbeit dar. Kapitel 4 erläutert den neuen Ansatz zur Modellierung von Signalwahrscheinlichkeiten durch quadratische Optimierung. Anschließend werden in Kapitel 5 experimentelle Ergebnisse dargestellt und mit existierenden Ansätzen verglichen, bevor im Kapitel 6 eine kurze Zusammenfassung die Arbeit abschließt.

\section{Definitionen}

Für die im vorherigen Kapitel beschriebenen Problemstellung sind in der Regel zwei Arten von Wahrscheinlichkeitsverteilungen entscheidend (Najm, 1994):

Stationäre Wahrscheinlichkeit: Die stationäre Wahrscheinlichkeit $P\left(x_{s}\right)$ eines Signals $x$ ist definiert als der durchschnittliche Anteil von Taktzyklen in denen der Signalwert entweder logisch Eins $\left(P\left(x_{s}=1\right)\right)$ bzw. logisch Null $\left(P\left(x_{s}=0\right)\right)$ ist. Damit ist $x_{s}$ Element der Menge $\{0,1\}$.

Schaltwahrscheinlichkeit: Die Schaltwahrscheinlichkeit $P\left(x_{t}\right)$ eines Signals $x$ ist definiert als der durchschnittliche Anteil von Taktzyklen in denen das Signal seinen Wert ändert $\left(P\left(x_{t}=s\right)\right)$ oder konstant bleibt $\left(P\left(x_{t}=k\right)\right)$. Damit ist $x_{t}$ Element der Menge $\{s($ chaltend $), k($ onstant $)\}$.

Im Weiteren wird folgende Notation für die Beschreibung von Wahrscheinlichkeitsverteilungen verwendet:

$P(a b c):=P(a \cap b \cap c)$

$P(\hat{a} \hat{b} \hat{c}):=P(a=\hat{a} \cap b=\hat{b} \cap c=\hat{c})$

$\hat{a}, \hat{b}, \hat{c} \in\{0,1, s, k\}$

Damit kennzeichnet $P(a b c)$ die allgemeine Verteilung und $P(\hat{a} \hat{b} \hat{c})$ einen expliziten Wert $\hat{a} \hat{b} \hat{c}$ dieser Verteilung. Um die Begrenzung der Wertemenge auf eine stationäre bzw. Schaltwahrscheinlichkeit zu kennzeichnen wird ein tiefergestelltes $s$ bzw. $t$ an die Variable angehängt:

$x_{s} \in\{0,1\} \quad x_{t} \in\{s, k\}$

Des Weiteren kann die gesamte Wahrscheinlichkeitsverteilung $P(a b c)$ auch als Vektor $\mathbf{P}(\mathbf{a b c})$ aufgefasst werden

$\boldsymbol{P}(\boldsymbol{a b c}):=\left[\begin{array}{c}P\left(\hat{a}_{0} \hat{b}_{0} \hat{c}_{0}\right) \\ P\left(\hat{a}_{0} \hat{b}_{0} \hat{c}_{1}\right) \\ \vdots \\ P\left(\hat{a}_{i} \hat{b}_{i} \hat{c}_{i}\right) \\ \vdots\end{array}\right] \quad \forall i$

wobei $a_{i}, b_{i}, c_{i}$ jeweils das i-te Element der Variablenmenge (z.B. $\{0,1\}$ ) bezeichnet.

Obwohl stationäre Wahrscheinlichkeit und Schaltwahrscheinlichkeit in der Regel als getrennte Wahrscheinlichkeiten angegeben werden sind ihre Werte nicht unabhängig voneinander. Für die Schaltwahrscheinlichkeiten eines Signals lässt sich allgemein zeigen, dass die Wahrscheinlichkeit eines steigenden Flanke gleich der Wahrscheinlichkeit einer fallenden Flanke ist (Najm, 1993):

$P\left(x_{t}=s \cap x_{s}=1\right)=P\left(x_{t}=s \cap x_{s}=0\right)$

Nimmt man zusätzlich die Definition der Randwahrscheinlichkeiten für die Verbundverteilung aus stationärer Wahrscheinlichkeit und Schaltwahrscheinlichkeit:

$$
\begin{aligned}
& P\left(x_{t}=s \cap x_{s}=1\right)+P\left(x_{t}=s \cap x_{s}=0\right)=P\left(x_{t}=s\right) \\
& P\left(x_{t}=k \cap x_{s}=1\right)+P\left(x_{t}=k \cap x_{s}=0\right)=P\left(x_{t}=k\right) \\
& P\left(x_{t}=s \cap x_{s}=0\right)+P\left(x_{t}=k \cap x_{s}=0\right)=P\left(x_{s}=0\right)
\end{aligned}
$$


$P\left(x_{t}=s \cap x_{s}=1\right)+P\left(x_{t}=k \cap x_{s}=1\right)=P\left(x_{s}=1\right)$

lässt sich durch Umformung mit Hilfe von (6) zeigen, dass

$P\left(x_{t}=s\right) \leq 2 \cdot \min \left(P\left(x_{s}=1\right), P\left(x_{s}=0\right)\right)$

gilt.

Damit ist die Schaltwahrscheinlichkeit eines Signals $x$ durch dessen stationäre Wahrscheinlichkeiten begrenzt.

\section{Existierende Ansätze}

Der in dieser Arbeit vorgestellte Ansatz gehört zur zweiten Unterklasse der iterativen Algorithmen. Dementsprechend wird im Folgenden ein kurzer Überblick über bereits existierende Arbeiten auf diesem Gebiet gegeben:

\subsection{Ansätze zur stationären Wahrscheinlichkeit}

Parker und McCluskey (1975) verwenden zur Berechnungen von Signalwahrscheinlichkeiten die Annahme, dass Signale grundsätzlich unabhängig sind. Damit lassen sich alle Signalabhängigkeiten auflösen und es gilt:

$P\left(\hat{x}_{s} \hat{y}_{s}\right)=P\left(\hat{x}_{s}\right) P\left(\hat{y}_{s}\right)$

Ercolani et al. (1989) definieren die Korrelation zwischen zwei Signalen $C$ :

$C\left(\hat{x}_{s} \hat{y}_{s}\right)=\frac{P\left(\hat{x}_{s} \hat{y}_{s}\right)}{P\left(\hat{x}_{s}\right) P\left(\hat{y}_{s}\right)}$

und benutzen diese zur Berechnung der Wahrscheinlichkeiten innerhalb der Schaltung. Korrelationen höherer Ordnung werden durch

$C\left(\hat{x}_{s} \hat{y}_{s} \hat{z}_{s}\right)=C\left(\hat{x}_{s} \hat{y}_{s}\right) \cdot C\left(\hat{x}_{s} \hat{z}_{s}\right) \cdot C\left(\hat{y}_{s} \hat{z}_{s}\right)$

unter der Annahme

$P\left(\hat{x}_{s} \hat{y}_{s} \hat{z}_{s}\right)=\frac{P\left(\hat{x}_{s} \hat{y}_{s}\right) \cdot P\left(\hat{y}_{s} \hat{z}_{s}\right) \cdot P\left(\hat{x}_{s} \hat{z}_{s}\right)}{P\left(\hat{x}_{s}\right) P\left(\hat{y}_{s}\right) P\left(\hat{z}_{s}\right)}$.

approximiert.

\subsection{Ansätze zur Schaltwahrscheinlichkeit}

Um die Schaltwahrscheinlichkeit abzuschätzen existieren ebenfalls mehrere Ansätze. Die erste Möglichkeit ist temporale Unabhängigkeit der einzelnen Signalzustände anzunehmen (Najm, 1994). Damit lässt sich die Schaltwahrscheinlichkeit durch die stationäre Wahrscheinlichkeit angeben:

$P\left(x_{t}=s\right)=2 \cdot P\left(x_{s}=0\right) \cdot P\left(x_{s}=1\right)$

Unter der Annahme von temporaler Abhängigkeit eines Signals zum vorherigen Taktzyklus wurde von Marculescu et al. (1994) die Definition von Ercolani et al. (1989) auf die Modellierung von Schaltwahrscheinlichkeiten erweitert. Hierbei sind die Variablen in (13) nicht mehr nur aus der Menge $\{0,1\}$ sondern alle aus der Menge $\{0,1, s, k\}$. Höherdimensionale Wahrscheinlichkeiten werden wieder entsprechend mit (14) approximiert.

\section{Modellierung von Signalwahrscheinlichkeiten durch quadratische Optimierung}

Das Signalmodell in dieser Arbeit geht von zweidimensionalen Wahrscheinlichkeiten $P(x y)$ aus. Außerdem wird angenommen, dass Schaltwahrscheinlichkeit und stationäre Wahrscheinlichkeit unabhängig sind. Damit unterteilt sich die Aufgabe zur Berechnung von Signalwahrscheinlichkeiten in die Approximation von dreidimensionalen Verbundwahrscheinlichkeiten auf Basis von bivariaten Verbundwahrscheinlichkeiten sowie Regeln für die iterative Berechnung.

\subsection{Signalmodell}

Aufgabe des Signalmodells ist es aus den bekannten Wahrscheinlichkeiten $P(\hat{x} \hat{y}), P(\hat{x} \hat{z})$ und $P(\hat{y} \hat{z})$ die Wahrscheinlichkeiten $\tilde{P}(\hat{x} \hat{y} \hat{z})$ einer approximativen dreidimensionalen Verbundverteilung zu konstruieren. Nimmt man hierbei an, dass Signale nur paarweise abhängig sind müssen folgende Gleichungen gelten:

$$
\begin{aligned}
& P(\hat{x} \hat{y} \hat{z})=P(\hat{x} \hat{y}) P(\hat{z}) \\
& P(\hat{x} \hat{y} \hat{z})=P(\hat{x} \hat{z}) P(\hat{y}) \\
& P(\hat{x} \hat{y} \hat{z})=P(\hat{y} \hat{z}) P(\hat{x})
\end{aligned}
$$

Da dies für beliebige Verbundverteilungen $P(x y), P(y z)$ und $P(x z)$ im Allgemeinen nicht gilt, wird die gesuchte Verteilung $P(x y z)$ wie folgt durch $\tilde{P}(x y z)$ approximiert:

$$
\begin{aligned}
& L_{1}(\hat{x} \hat{y} \hat{z})=\tilde{P}(\hat{x} \hat{y} \hat{z})-P(\hat{x} \hat{y}) P(\hat{z}) \\
& L_{2}(\hat{x} \hat{y} \hat{z})=\tilde{P}(\hat{x} \hat{y} \hat{z})-P(\hat{x} \hat{z}) P(\hat{y}) \\
& L_{3}(\hat{x} \hat{y} \hat{z})=\tilde{P}(\hat{x} \hat{y} \hat{z})-P(\hat{y} \hat{z}) P(\hat{x})
\end{aligned}
$$

$\tilde{\mathbf{P}}(\mathbf{x y z}):=\min _{\tilde{\boldsymbol{P}}(\boldsymbol{x}, \boldsymbol{y}, \boldsymbol{z})} \sum_{i=1}^{3} \sum_{(\hat{x} \hat{y} \hat{z}) \in \mathbb{M}^{3}}\left(L_{i}(\hat{x} \hat{y} \hat{z})\right)^{2}$

Hierbei geben (20) - (22) den Approximationsfehler der einzelnen Werte der Verteilung $\tilde{P}$ für die Annahmen (17) - (19) an. Gleichung 23 minimiert dann den summierten quadratischen Gesamtfehler aller einzelnen Werte der Verteilung $\tilde{P}$ gegenüber diesen Annahmen. Die Menge $\mathbb{M}$ bezeichnet hierbei die Variablenmenge, d.h. $\{0,1\}$ oder $\{s, k\}$.

Um sicherzustellen, dass die Verteilung $\tilde{P}(x y z)$ eine Wahrscheinlichkeitsverteilung darstellt, muss zusätzlich gelten:

$$
\begin{aligned}
0 \leq \tilde{P}(\hat{x} \hat{y} \hat{z}) \leq 1 & \forall \hat{x} \hat{y} \hat{z} \in \mathbb{M}^{3} \\
\sum_{\hat{x} \in \mathbb{M}} \tilde{P}(\hat{x} \hat{y} \hat{z})=P(\hat{y} \hat{z}) & \forall \hat{y} \hat{z} \in \mathbb{M}^{2} \\
\sum_{\hat{y} \in \mathbb{M}} \tilde{P}(\hat{x} \hat{y} \hat{z})=P(\hat{x} \hat{z}) & \forall \hat{x} \hat{z} \in \mathbb{M}^{2} \\
\sum_{\hat{z} \in \mathbb{M}} \tilde{P}(\hat{x} \hat{y} \hat{z})=P(\hat{x} \hat{y}) & \forall \hat{x} \hat{y} \in \mathbb{M}^{2}
\end{aligned}
$$




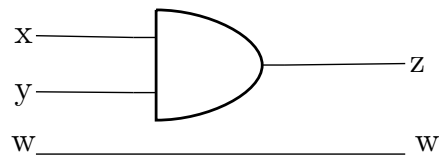

Abbildung 1. UND-Gatter mit abhängigem zusätzlichem Signal $w$

Da jede Variable $\hat{x}, \hat{y}$ und $\hat{z}$ aus einer zweiwertigen Menge $\mathbb{M}$ (entweder $\{0,1\}$ oder $\{s, k\}$ ) stammt geben die Gleichungen (25) - (27) insgesamt 12 Gleichheitsnebenbedingungen für das Optimierungsproblem aus (23). Hiervon sind $7 \mathrm{Be}-$ dingungen linear unabhängig.

Durch Wahl einer bestimmten Wahrscheinlichkeit $\tilde{P}\left(\hat{x}_{0} \hat{y}_{0} \hat{z}_{0}\right)$ (z.B.: $\hat{x}_{0} \hat{y}_{0} \hat{z}_{0}=000$ ) lassen sich durch diese Nebenbedingungen alle anderen Wahrscheinlichkeiten durch diese eine ausdrücken. Damit gilt für alle Wahrscheinlichkeiten aus $\tilde{P}(x y z)$ :

$\tilde{P}(\hat{x} \hat{y} \hat{z})=a \cdot \tilde{P}\left(\hat{x}_{0} \hat{y}_{0} \hat{z}_{0}\right)+b \quad \forall \hat{x} \hat{y} \hat{z} \in \mathbb{M}^{3}$

Die Parameter $a$ und $b$ ergeben sich hierbei aus der Lösung des linearen Gleichungssystems (25) - (27) und sind für jede Wahrscheinlichkeit $\tilde{P}(\hat{x} \hat{y} \hat{z})$ individuell. Zum Beispiel lässt sich $\tilde{P}\left(\hat{x}_{0} \hat{y}_{0} \hat{z}_{1}=001\right)$ folgendermaßen ausdrücken, wie sich leicht anhand von Gleichung (27) nachvollziehen lässt:

$\tilde{P}\left(\hat{x}_{0} \hat{y}_{0} \hat{z}_{1}\right)=\underbrace{P\left(\hat{x}_{0} \hat{y}_{0}\right)}_{=b}+(\underbrace{-1}_{=a} \cdot \tilde{P}\left(\hat{x}_{0} \hat{y}_{0} \hat{z}_{0}\right))$

Mit Hilfe der sieben nicht-trivialen Gleichheitsbeziehungen aus (28) lässt sich das Optimierungsproblem in (23) in ein eindimensionales Optimierungsproblem transformieren. Genauso lassen sich neue Grenzen $m$ und $n$ für die noch unbekannte Wahrscheinlichkeit $\tilde{P}\left(\hat{x}_{0} \hat{y}_{0} \hat{z}_{0}\right)$ angeben durch Einsetzen von (28) in (24):

$$
\begin{array}{r}
\min _{\tilde{P}\left(\hat{x}_{0}, \hat{y}_{0}, \hat{z}_{0}\right)}\left(d \tilde{P}^{2}\left(\hat{x}_{0} \hat{y}_{0} \hat{z}_{0}\right)+e \tilde{P}\left(\hat{x}_{0} \hat{y}_{0} \hat{z}_{0}\right)+f\right) \\
m \leq \tilde{P}\left(\hat{x}_{0} \hat{y}_{0} \hat{z}_{0}\right) \leq n
\end{array}
$$

Hierbei sind die Parameter $d, e, f, m$ und $n$ wiederum feste Funktionen der bereits bekannten zweidimensionalen Wahrscheinlichkeiten $P(\hat{x} \hat{y}), P(\hat{y} \hat{z})$ und $P(\hat{x} \hat{z})$.

Löst man nun das eindimensionale Optimierungsproblem in (30) unter der Nebenbedingung (31), so sind alle vorher geforderten Nebenbedingungen automatisch erfüllt und alle verbleibenden Wahrscheinlichkeiten lassen sich durch (28) berechnen.

Die Lösung von (30) lässt sich direkt angeben zu:

$P\left(\hat{x}_{0} \hat{y}_{0} \hat{z}_{0}\right)= \begin{cases}-\frac{e}{2 d}, & \text { falls } m \leq-\frac{e}{2 d} \leq n \\ m, & \text { falls } m>-\frac{e}{2 d} \\ n & , \text { falls } n<-\frac{e}{2 d}\end{cases}$

Hierdurch lässt sich die Approximation effizient durch explizite Formeln implementieren ohne die Notwendigkeit kostspieliger generischer Optimierungsverfahren.

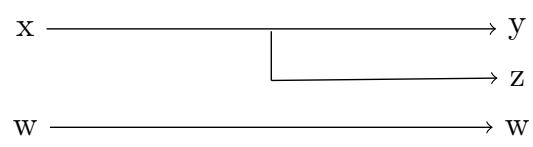

Abbildung 2. Signalverzweigung in einer kombinatorischen Schaltung

\subsection{Iterative Berechnung}

Mit Hilfe der vorgestellten Approximation im vorherigen Absatz lassen sich Regeln zur iterativen Berechnung von $\mathrm{Si}$ gnalwahrscheinlichkeiten aufstellen.

\subsubsection{Stationäre Wahrscheinlichkeiten}

Für eine Logikzelle mit 2 Eingängen und einem Ausgang lässt sich die stationäre Wahrscheinlichkeit an ihrem Ausgang auf Basis ihrer Logikfunktion berechnen (Parker und McCluskey, 1975). Abbildung 1 zeigt ein UND-Gatter mit den Eingängen $x$ und $y$, sowie ein drittes abhängiges Signal $w$. Aufgabe des Algorithmus ist es nun die Verbundwahrscheinlichkeit $P(w z)$ auf Basis der bekannten Verbundwahrscheinlichkeiten $P(x y), P(y w)$ und $P(x w)$ zu berechnen.

Diese Aufgabe lässt sich generell in zwei Teilaufgaben unterteilen:

1. Approximation der Verbundverteilung $\tilde{P}(x y w)$

2. Transformation von $\tilde{P}(x y w)$ zu $P(z w)$

Die Methode zur Approximation der Verbundverteilung $\tilde{P}(x y w)$ wurde bereits in Kapitel 4.1 vorgestellt. Mit Hilfe dieser Verbundverteilung kann die Verteilung $P(z w)$ dann wie folgt berechnet werden:

$$
\begin{aligned}
P(z=0 \cap w=\hat{w})= & \tilde{P}(x=0 \cap y=0 \cap w=\hat{w}) \\
& +\tilde{P}(x=0 \cap y=1 \cap w=\hat{w}) \\
& +\tilde{P}(x=1 \cap y=0 \cap w=\hat{w})
\end{aligned}
$$

$P(z=1 \cap w=\hat{w})=\tilde{P}(x=1 \cap y=1 \cap w=\hat{w})$

Für Zellen mit anderen Funktionen wie z.B. ODER, NICHT, XOR oder NAND lassen sich ebenso Regeln aufstellen.

Jede Signalverzweigung in der Schaltung (siehe Abbildung 2) bei der aus einem Signal $x$ zwei neue Signale $y$ und $z$ generiert werden führt eine zusätzliche Verbundwahrscheinlichkeit $P(y z)$, sowie entsprechende Verbundwahrscheinlichkeiten $P(y w)$ und $P(z w)$ zu weiteren abhängigen Signalen, ein:

$P(y=0 \cap z=0)=P(x=0)$

$P(y=1 \cap z=1)=P(x=1)$ 
$P(y=0 \cap z=1)=P(y=1 \cap z=0)=0$

$P(y w)=P(z w)=P(x w)$

Hierdurch können durch iterative Verarbeitung alle stationären Signalwahrscheinlichkeiten innerhalb der Schaltung aus den Verbundwahrscheinlichkeiten der Eingänge berechnet werden. Zellen mit mehr als 2 Eingängen können hierbei durch eine Verkettung mehrerer zweistelliger Logikfunktionen integriert werden.

\subsubsection{Schaltwahrscheinlichkeiten}

Die Berechnung von Schaltwahrscheinlichkeiten folgt prinzipiell den selben Regeln wie die von stationären Wahrscheinlichkeiten. Am Beispiel des UND-Gatters aus Abbildung 1 lassen sich hier z.B. folgende Formeln aufstellen:

$$
\begin{aligned}
& P(z=s \cap w=\hat{w})=\tilde{P}(x=s \cap y=k \cap w=\hat{w}) \cdot P(y=1) \\
& \quad+\tilde{P}(x=k \cap y=s \cap w=\hat{w}) \cdot P(x=1) \\
& \quad+\tilde{P}(x=s \cap y=s \cap w=\hat{w}) \cdot P(x=y)
\end{aligned}
$$

$$
P(z=k \cap w=\hat{w})=P(w=\hat{w})-P(z=s \cap w=\hat{w})
$$

Wie sich in (39) erkennen lässt, gehen in die Berechnung der Schaltwahrscheinlichkeiten die stationären Wahrscheinlichkeiten ein, da die Signalwerte an den Gattereingängen bestimmen ob sich ein schaltendes Signal zum Ausgang propagieren kann. In (39) stellen die ersten beiden Summanden die Wahrscheinlichkeiten dar, dass nur einer der beiden Eingänge schaltet, und der dritte Summand, dass beide Eingänge gleichzeitig schalten.

Da bei der Berechnung der Schaltwahrscheinlichkeit angenommen wurde, dass diese von den stationären Wahrscheinlichkeiten unabhängig ist, muss anschließend die berechnete Verbundwahrscheinlichkeit $P(z w)$ mit Gleichung 11 begrenzt werden.

\section{Experimentelle Ergebnisse}

\subsection{Kostenfunktionen zur Bewertung der Approximati- onsgüte}

Um die vorgestellte Methode zu bewerten wird eine Funktion benötigt, welche die Approximationsqualität bewertet. Für eine approximierte Verteilung $\tilde{P}(x y z)$ kann für jedes Belegungstupel $\hat{x} \hat{y} \hat{z}$ der approximierte Wert dem Wert der realen Verteilung $P(x y z)$ gegenübergestellt werden. Da die Wahrscheinlichkeitsverteilung $P(x y z)$ auch als Vektor $\boldsymbol{P}(\boldsymbol{x} \boldsymbol{y} z)$ aufgefasst werden kann (siehe Gl. 5) lässt sich die Approximationsgüte durch die Distanz der beiden Vektoren $\tilde{\boldsymbol{P}}(\boldsymbol{x} \boldsymbol{y} z)$ und $\boldsymbol{P}(\boldsymbol{x} \boldsymbol{y} z)$ abschätzen.
Hierbei bietet sich insbesondere die TschebyscheffDistanz $d_{\infty}$ an:

$d_{\infty}=\max |P(x y z)-\tilde{P}(x y z)|$

Die Tschebyscheff-Distanz gibt somit denjenigen Einzelwert der Verteilung an, für den der größte Approximationsfehler gemacht wird. Da die Vektoren Wahrscheinlichkeitsverteilungen darstellen, liegt die Tschebyscheff-Distanz zwischen 0 und 1, wobei 0 eine perfekte Approximation und 1 dementsprechend die schlechtest mögliche Approximation darstellt.

$0 \leq d_{\infty} \leq 1$

Da diese Approximation im Zuge der Berechnung über mehrere verschiedene Signale gemacht werden muss, ergibt sich für jedes Signaltripel $x^{(i)} y^{(i)} z^{(i)}$ ein Distanzwert $d_{\infty}^{(i)}$. Somit muss zur globalen Bewertung aus diesen Einzelwerten wieder ein Gesamtwert gebildet werden. Hierbei werden in dieser Arbeit der Mittelwert sowie das Maximum über alle ausgerechneten Distanzen genommen. Dies gibt dementsprechend den mittleren Approximationsfehler, sowie den maximalen Approximationsfehler, über alle Signale an.

\subsection{Test anhand eines Mikroprozessors}

Zum Test des vorgestellten Modells und zum Vergleich mit existierenden Ansätzen wurden verschiedene Programme auf einem Instruktionssatzsimulator für einen $16 \mathrm{Bit} \mathrm{Mi}$ kroprozessor ausgeführt und auftretende Signale für jeweils 2000000 Taktzyklen extrahiert. Hieraus wurden dann entsprechend die realen stationären Wahrscheinlichkeiten und Schaltwahrscheinlichkeiten der Signale berechnet. Abbildung 3 stellt hierbei die Approximationsgüte des vorgestellten Ansatzes denen existierender Ansätze für verschiedene Programme gegenüber.

Wie zu sehen ist, stellt die vorgestellte Methode einen wesentlichen Genauigkeitszuwachs bei Verwendung realistischer Signalwahrscheinlichkeiten dar. Dies ist vor allem auf die Herleitung der Approximation basierend auf den Annahmen (17) - (19) zurückzuführen.

Um die Laufzeiteigenschaften des Algorithmus zu testen und denen existierender Ansätze gegenüberzustellen, wurden zusätzlich Schaltungen der ISCAS (Brglez, 1985) und ITC (Davidson, 1999) Benchmarksammlungen verwendet. Abbildung 4 zeigt die entsprechenden Laufzeiten im Vergleich mit anderen Algorithmen über der Zahl der in der Schaltung enthaltenen Gatter.

Die Laufzeit ist hierbei nicht nur von der Gatteranzahl funktional abhängig, sondern im Wesentlichen auch von der Schaltungstopologie bestimmt. Im Vergleich mit dem Ansatz nach Ercolani et al. (1989) hat der vorgestellte Ansatz ähnliche Laufzeiten. 


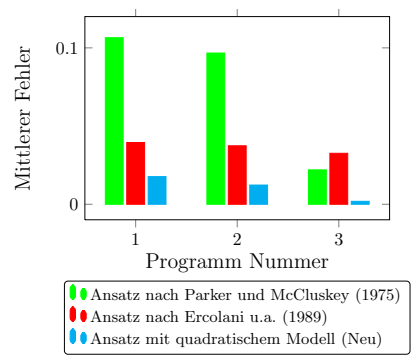

(a) Mittlerer Fehler (stationäre Wahrscheinlichkeit)

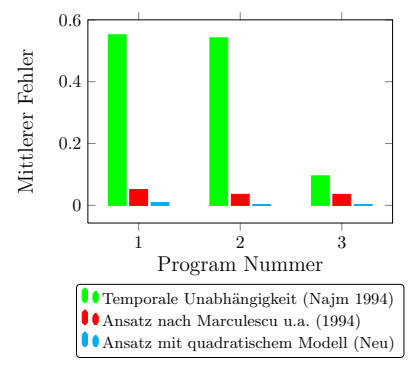

(c) Mittlerer Fehler (Schaltwahrscheinlichkeit)

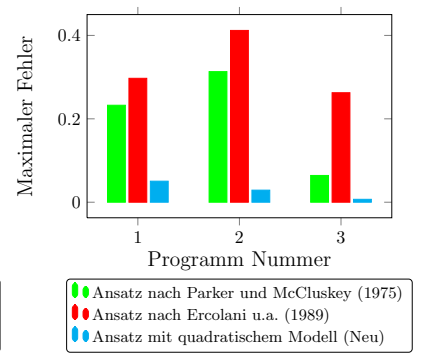

(b) Max. Fehler (stationäre Wahrscheinlichkeit)

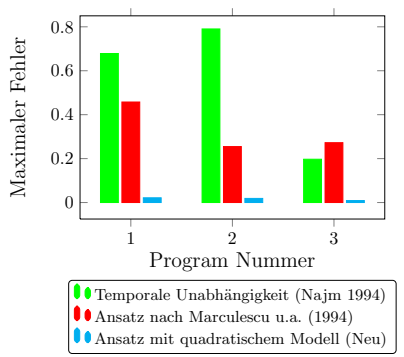

(d) Max. Fehler (Schaltwahrscheinlichkeit)

Abbildung 3. Vergleich der Approximationsgüte

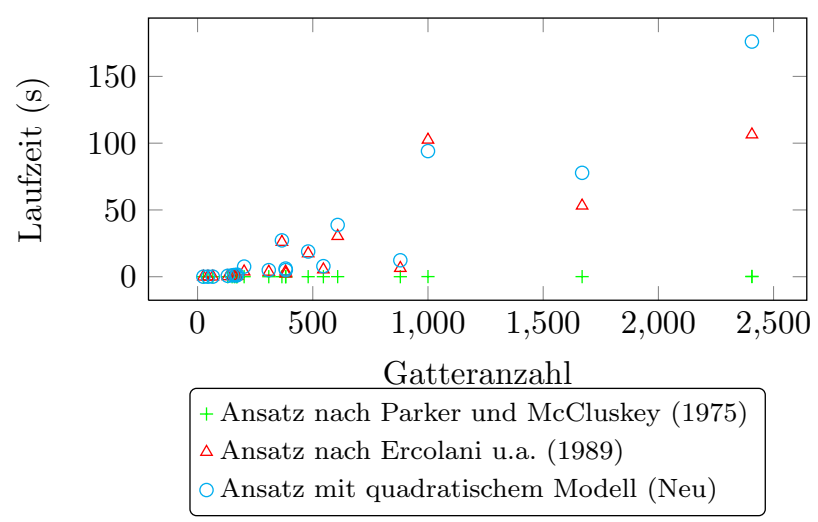

Abbildung 4. Laufzeit der Algorithmen in Abhängigkeit der Gatterzahl

\section{Diskussion}

In dieser Arbeit wurde ein neuer Ansatz zur Modellierung von Signalwahrscheinlichkeiten vorgestellt. Der Ansatz basiert auf der Approximation von Signalwahrscheinlichkeiten auf Basis eines quadratischen Modells. Das quadratische Problem lässt sich analytisch lösen und somit effizient implementieren.

Beim Vergleich mit anderen Ansätzen zeigt sich ein deutlicher Genauigkeitszuwachs bei der Verwendung von realistischen Signalwahrscheinlichkeiten. Die Laufzeit ist ähnlich der existierender Ansätze gleicher Komplexität.

Danksagung. Diese Arbeit wurde von der Deutschen Forschungsgemeinschaft (DFG) im Rahmen des Schwerpunktprogramms "Entwurf und Architekturen verlässlicher eingebetteter Systeme" unterstützt (SPP 1500 - spp1500.itec.kit.edu).

\section{Literatur}

Brglez, F.: Neutral netlist of ten combinational benchmark circuits and a target translator in FORTRAN, in: IEEE International Symposium on Circuits and Systems (ISCAS), 1985.

Chen, L. und Tahoori, M. B.: An efficient probability framework for error propagation and correlation estimation, in: IEEE International On-Line Testing Symposium (IOLTS), 2012.

Davidson, S.: ITC'99 Benchmark Circuits - Preliminary Results, in: IEEE International Test Conference (ITC), 1999.

Ercolani, S., Favalli, M., Damiani, M., Olivo, P. und Ricco, B.: Estimate of signal probability in combinational logic networks, in: IEEE European Test Conference (ETC), 1989.

Lorenz, D., Georgakos, G. und Schlichtmann, U.: Aging analysis of circuit timing considering NBTI and HCI, in: IEEE International On-Line Testing Symposium (IOLTS), 2009.

Marculescu, R., Marculescu, D. und Pedram, M.: Switching activity analysis considering spatiotemporal correlations, in: IEEE International Conference on Computer-Aided Design (ICCAD), 1994.

Najm, F. N.: Transition density: a new measure of activity in digital circuits, IEEE Transactions on Computer-Aided Design of Integrated Circuits and Systems (TCAD), 12, 310-323, 1993.

Najm, F. N.: A survey of power estimation techniques in VLSI circuits, IEEE Transactions on Very Large Scale Integration (VLSI) Systems, 2, 446-455, 1994.

Nassif, S. R.: Power Grid Analysis Benchmarks, in: IEEE Asia and South Pacific Design Automation Conference (ASPDAC), 2008.

Obermeier, B. und Johannes, F.: Temperature-aware global placement, in: IEEE Asia and South Pacific Design Automation Conference (ASPDAC), 143-148, 2004.

Parker, K. und McCluskey, E.: Probabilistic treatment of general combinational networks, IEEE Transactions on Computers, 100, 668-670, 1975.

Schneider, P., Schlichtmann, U. und Wurth, B.: Fast power estimation of large circuits, IEEE Design \& Test of Computers, 13, 70$78,1996$. 